A survey was sent to 600 pediatricians. Three hundred eighty (68\%) of the 563 pediatricians who were located responded to the survey. Of these 380 pediatricians, 279 provided routine immunizations to children. Of the 270 pediatricians who vaccinated children with hepatitis $B$ vaccine and indicated their practice regarding the birth dose, $50 \%$ offered the first dose of hepatitis B vaccine at birth to all infants; the rest either offered the vaccine at birth only to infants of hepatitis B surface antigen (HBsAg)-positive mothers and mothers whose serostatus is unknown, or did not offer the birth dose to any infants. Practicing in the inner city, working for a medical school or government hospital, and living in a state with universal immunization supply policies were associated with the respondent's giving the birth dose. The strongest perceived barriers to giving the birth dose in the hospital were the difficulty tracking these vaccines (39\%), the increased cost (27\%), and the lack of reimbursement from insurance companies (26\%). If a combination vaccine that includes hepatitis $\mathrm{B}$; diphtheria, tetanus, and pertussis (diphtheria and tetanus toxoids and acellular pertussis vaccine); and polio (inactivated poliovirus vaccine) antigens become available in the near future, then $38 \%$ of physicians who currently give the birth dose to all infants would prefer to wait until 2 months of age to initiate hepatitis B immunization.

According to the researchers, this study demonstrates that most pediatricians are abiding by current recommendations, which fits the model of physician agreement before adoption. However, as new vaccines are introduced into the mandated childhood immunization program, physicians will trend toward combination vaccines and delaying administering the hepatitis $B$ vaccine until 1 to 2 months of age. The delay in immunization will necessitate the early and focused identification of HBsAg-positive pregnant women to ensure that these particular infants are immunized at birth. Finding methods to improve the universal screening of women for $\mathrm{HBsAg}$ is warranted, because, in 1993 , only $22 \%$ of infants born to mothers of unknown HBsAg status received the birth dose of HBV vaccine.

FROM: Cooper A, Yusuf H, Rodewald L, Malik T, Pollard R, Pickering L. Attitudes, practices, and preferences of pediatricians regarding initiation of hepatitis $B$ immunization at birth. Pediatrics 2001;108:E98.

\section{Epidemiology of Community-Acquired MRSA in Minnesota}

Naimi et al. from the Minnesota Department of Health reviewed records from 10 Minnesota health facilities to identify cases of methicillin-resistant Staphylococcus aureus (MRSA) infection that occurred from 1996 to 1998 and to identify which cases were community acquired. Susceptibility testing and pulsed-field gel electrophoresis (PFGE) subtyping were performed on available isolates. A total of 354 patients (median age, 16 years) with community-acquired MRSA infection were identified. Most case patients (299 [84\%]) had skin infec- tions, and 103 (29\%) were hospitalized. More than $90 \%$ of isolates were susceptible to all antimicrobial agents tested, with the exception of beta-lactams and erythromycin. Of 334 patients treated with antimicrobial agents, 282 $(84 \%)$ initially were treated with agents to which their isolates were nonsusceptible. Of 174 Minnesota isolates tested, 150 (86\%) belonged to one PFGE clonal group. Community-acquired MRSA infections were identified throughout Minnesota. Although most isolates were genetically related and susceptible to multiple antimicrobials, they were generally nonsusceptible to initial empirical therapy.

FROM: Naimi TS, LeDell KH, Boxrud DJ, et al. Epidemiology and clonality of community-acquired methicillin-resistant Staphylococcus aureus in Minnesota, 19961998. Clin Infect Dis 2001;33:990-996.

\section{Perioperative Prophylaxis With Vancomycin May Be Cost-Effective for Coronary Artery Bypass Graft Surgery}

Routine use of vancomycin for perioperative prophylaxis is discouraged, principally to minimize microbial resistance to it. However, outcomes and costs of this recommendation have not been assessed. Zanetti et al. from Channing Laboratory, Brigham and Women's Hospital, used decision-analytic models to compare clinical results and cost-effectiveness of no prophylaxis, cefazolin, and vancomycin in coronary artery bypass graft surgery. They focused on patients who underwent coronary artery bypass graft surgery because this is a large, relatively homogeneous population with substantial risk for serious surgical-site infection.

In the base case, vancomycin resulted in $7 \%$ fewer surgical-site infections and $1 \%$ lower all-cause mortality and saved $\$ 117$ per procedure, compared with cefazolin. In turn, cefazolin resulted in substantially fewer infections and deaths and lower costs than no prophylaxis.

The authors concluded that perioperative antibiotic prophylaxis with vancomycin is usually more effective and less expensive than perioperative antibiotic prophylaxis with cefazolin. Data on vancomycin's impact on resistance are needed to quantify the trade-off between individual patients' improved clinical outcomes and lower costs and the future long-term consequences to society.

FROM: Zanetti G, Goldie SJ, Platt R. Clinical consequences and cost of limiting use of vancomycin for perioperative prophylaxis: example of coronary artery bypass surgery. Emerg Infect Dis 2001;7:820-827.

\section{Surveillance of Postpartum Infections Using Automated HMO Data}

The epidemiology of postpartum infections has not been well characterized. In part, this is because of the limitations of surveillance systems, which usually monitor infections that are recognized during hospitalization. 